\title{
IRREDUCIBLE SUBGROUPS OF ALGEBRAIC GROUPS
}

\author{
by MARTIN W. LIEBECK ${ }^{\dagger}$ \\ (Department of Mathematics, Imperial College, London SW7 2BZ) \\ and DONNA M. TESTERMAN \\ (Department of Mathematics, University of Lausanne, Switzerland)
}

[Received 27 February 2003. Revised 18 July 2003]

\begin{abstract}
A closed subgroup of a semisimple algebraic group $G$ is said to be $G$-irreducible if it lies in no proper parabolic subgroup of $G$. We prove a number of results concerning such subgroups. Firstly they have only finitely many overgroups in $G$; secondly, with some specified exceptions, there exist $G$-irreducible subgroups of type $A_{1}$; and thirdly, we prove an embedding theorem for $G$-irreducible subgroups.
\end{abstract}

\section{Introduction}

Let $G$ be a semisimple algebraic group over an algebraically closed field $K$ of characteristic $p \geqslant 0$. Following Serre, we define a subgroup $\Gamma$ of $G$ to be $G$-irreducible if $\Gamma$ is closed, and lies in no proper parabolic subgroup of $G$. When $G=S L(V)$, this definition coincides with the usual notion of irreducibility on $V$. The definition follows the philosophy, developed over the years by Serre, Tits and others, of generalizing standard notions of representation theory (morphisms $\Gamma \rightarrow S L(V)$ ) to situations where the target group is an arbitrary semisimple algebraic group. For an exposition, see for example [8, Part II].

In this paper we study the collection of connected $G$-irreducible subgroups of semisimple algebraic groups $G$. Our first theorem is a finiteness result, showing that connected $G$-irreducible subgroups are 'nearly maximal'.

THEOREM 1 Let $G$ be a connected semisimple algebraic group, and let $A$ be a connected $G$-irreducible subgroup of $G$. Then $A$ is contained in only finitely many subgroups of $G$.

Since connected $G$-irreducible subgroups are necessarily semisimple (see Lemma 2.1), the smallest possibility for such a subgroup is $A_{1}$. The next result shows that $G$-irreducible $A_{1}$ subgroups usually exist. In large characteristic this is hardly surprising, as maximal $A_{1}$ subgroups usually exist; but in low characteristic maximal $A_{1}$ subgroups do not exist (see [5]), and the result provides a supply of nearly maximal $A_{1}$ subgroups.

THEOREM 2 Let $G$ be a simple algebraic group over $K$. If $G=A_{n}$, assume that $p>n$ or $p=0$. Then $G$ has a $G$-irreducible subgroup of type $A_{1}$.

\footnotetext{
${ }^{\dagger}$ E-mail: M.Liebeck@imperial.ac.uk

"E-mail: donna.testerman@epfl.ch
}

Quart. J. Math. 55 (2004), 47-55; doi: 10.1093/qmath/hag042

Quart. J. Math. Vol. 55 Part 1 (c) Oxford University Press 2004; all rights reserved 
In the excluded case $G=A_{n}, 0<p \leqslant n$, it is easy to see that an irreducible subgroup $A_{1}$ exists if and only if all prime factors of $n+1$ are at most $p$.

In a subsequent paper [6] we shall use the $G$-irreducible $A_{1}$ s constructed in the proof of Theorem 2 to exhibit examples of epimorphic subgroups of minimal dimension in simple algebraic groups, as defined in [2]. (A closed subgroup $H$ of the connected algebraic group $G$ is said to be epimorphic if any morphism of $G$ into an algebraic group is determined by its restriction to $H$. [2, Theorem 1] has a number of equivalent formulations of this definition: for example, $H$ is epimorphic if and only if, whenever $V$ is a rational $G$-module and $V \downarrow H=X \oplus Y$, then $X, Y$ are $G$-invariant.)

Our final theorem concerns the description of conjugacy classes of connected $G$-irreducible subgroups of semisimple algebraic groups $G$. When $G$ is simple, it has only finitely many classes of maximal connected subgroups (see [5, Corollary 3]). This is in general not the case for connected $G$-irreducible subgroups (see for example Corollary 4.5 below). However, Theorem 3 below shows that there is a finite collection of conjugacy classes of closed connected subgroups such that every $G$-irreducible subgroup is embedded in a specified way in a member of one of these classes. For the precise statement we require the following definition.

DEFINITION Let $X, Y$ be connected linear algebraic groups over $K$.

(i) Suppose $X$ is simple. We say $X$ is a twisted diagonal subgroup of $Y$ if $Y=Y_{1} \ldots Y_{t}$, a commuting product of simple groups $Y_{i}$ of the same type as $X$, and if each projection $X \rightarrow Y_{i} / Z\left(Y_{i}\right)$ is non-trivial and involves a different Frobenius twist.

(ii) More generally, if $X$ is semisimple, say $X=X_{1} \ldots X_{r}$ with each $X_{i}$ simple, we say $X$ is a twisted diagonal subgroup of $Y$ if $Y=Z_{1} \ldots Z_{r}$, a commuting product of semisimple subgroups $Z_{i}$, and, writing $\bar{X}=X / Z(X)=\bar{X}_{1} \ldots \bar{X}_{r}$ and $\bar{Y}=Y / Z(Y)=\bar{Z}_{1} \ldots \bar{Z}_{r}$, each $\bar{X}_{i}$ is a twisted diagonal subgroup of $\bar{Z}_{i}$.

THEOREM 3 Let $G$ be a connected semisimple algebraic group of rank $l$. Then there is a finite set $\mathcal{C}$ of conjugacy classes of connected semisimple subgroups of $G$, of size depending only on $l$, with the following property. If $X$ is any connected $G$-irreducible subgroup of $G$, then there is a subgroup $Y \in \bigcup \mathcal{C}$ such that $X$ is a twisted diagonal subgroup of $Y$.

The above results concern connected $G$-irreducible subgroups. Examples of non-connected $G$ irreducible subgroups $X$ such that $X^{0}$ is not $G$-irreducible are easy to come by: for instance, $X=$ $N_{G}(T)$, the normalizer of a maximal torus $T$ is such an example, and there are many others for which $C_{G}\left(X^{0}\right)$ contains a non-trivial torus. However, we have not found any examples for which $C_{G}\left(X^{0}\right)$ contains no non-trivial torus. It may be the case that if $X$ is a non-connected $G$-irreducible subgroup such that $X^{0}$ is not $G$-irreducible, then $C_{G}\left(X^{0}\right)$ necessarily contains a non-trivial torus; this is easily seen to be true when $G=A_{n}$.

NotATION For $G$ a simple algebraic group over $K$ and $\lambda$ a dominant weight, we denote by $V_{G}(\lambda)$ (or just $\lambda$ ) the rational irreducible $K G$-module of high weight $\lambda$. When $p>0$, the irreducible module $\lambda$ twisted by a $p^{r}$-power field morphism of $G$ is denoted by $\lambda^{\left(p^{r}\right)}$. Finally, if $V_{1}, \ldots, V_{k}$ are $X$-modules then $V_{1} / \ldots / V_{k}$ denotes a $G$-module having the same composition factors as $V_{1} \oplus \ldots \oplus V_{k}$. 


\section{Preliminaries}

As above, let $G$ be a semisimple connected algebraic group over the algebraically closed field $K$ of characteristic $p$. We begin with two elementary results concerning $G$-irreducible subgroups.

LEMMA 2.1 If $X$ is a connected $G$-irreducible subgroup of $G$, then $X$ is semisimple, and $C_{G}(X)$ is finite.

Proof. Suppose $C=C_{G}(X)^{0} \neq 1$. If $C$ contains a non-trivial torus $T$, then $X \leqslant C_{G}(T)$, which lies in a parabolic; otherwise $C$ is unipotent, so $X \leqslant N_{G}(C)$ which lies in a parabolic by [3]. In either case we have a contradiction, and so $C_{G}(X)^{0}=1$, giving the result.

Lemma 2.2 Suppose $G$ is classical, with natural module $V=V_{G}\left(\lambda_{1}\right)$. Let $X$ be a semisimple connected closed subgroup of $G$. If $X$ is $G$-irreducible then one of the following holds:

(i) $G=A_{n}$ and $X$ is irreducible on $V$;

(ii) $G=B_{n}, C_{n}$ or $D_{n}$ and $V \downarrow X=V_{1} \perp \ldots \perp V_{k}$ with the $V_{i}$ all non-degenerate, irreducible and inequivalent as $X$-modules;

(iii) $G=D_{n}, p=2$, X fixes a non-singular vector $v \in V$, and $X$ is a $G_{v}$-irreducible subgroup of $G_{v}=B_{n-1}$.

Proof. Part (i) is clear, so assume $G=S p(V)$ or $S O(V)$. Let $W$ be a minimal non-zero $X$-invariant subspace of $V$. Then $W$ is either non-degenerate or totally isotropic. In the first case induction gives a non-degenerate decomposition as in (ii); note that no two of the $V_{i}$ are equivalent as $X$-modules since otherwise, if say $V_{1} \downarrow X \cong V_{2} \downarrow X$ via an isometry $\phi: V_{1} \rightarrow V_{2}$, then $X$ fixes the diagonal totally singular subspace $\left\{v+i \phi(v): v \in V_{1}\right\}$ of $V_{1}+V_{2}$ (where $i^{2}=-1$ ), hence lies in a parabolic. Finally, if $W$ is totally isotropic it can have no non-zero singular vectors (as $X$ does not lie in a parabolic), so we must have $G=S O(V)$ with $p=2$ and $W=\langle v\rangle$ non-singular, yielding (iii).

The next result is fairly elementary for classical groups $G$, but rests on the full weight of the memoirs $[\mathbf{5}, \mathbf{7}]$ for exceptional groups.

PROPOSITION 2.3 [5, Corollary 3] If $G$ is a simple algebraic group then $G$ has only finitely many conjugacy classes of maximal closed subgroups of positive dimension. The number of conjugacy classes is bounded in terms of the rank of $G$.

We shall also require a description of the maximal closed connected subgroups of semisimple algebraic groups. Let $G$ be a semisimple algebraic group, and write $G=G_{1} \cdots G_{r}$, a commuting product of simple factors $G_{i}$. Define $\mathcal{M}(G)$ to be the following set of connected subgroups of $G$ :

(1) for $j \in\{1, \ldots, r\}$, subgroups $\left(\Pi_{i \neq j} G_{i}\right) \cdot M_{j}$, with $M_{j}$ a maximal connected proper subgroup of $G_{j}$, and

(2) for $r \geqslant 2$ and distinct $j, k \in\{1, \ldots, r\}$ such that there is a surjective morphism $\phi: G_{j} \rightarrow G_{k}$, subgroups of the form

$$
G_{j, k}(\phi)=\left(\Pi_{i \neq j, k} G_{i}\right) \cdot D_{j, k},
$$

where $D_{j, k}=\left\{(g, \phi(g)): g \in G_{j}\right\}$, a closed connected diagonal subgroup of $G_{j} G_{k}$. 
LEMMA 2.4 The collection $\mathcal{M}(G)$ comprises all the maximal closed connected subgroups of the semisimple group $G$.

Proof. It is clear that the members of $\mathcal{M}(G)$ are maximal closed connected subgroups of $G$. Conversely, suppose that $M$ is a maximal closed connected subgroup of $G$. Factoring out $Z(G)$, we may assume that $Z(G)=1$. Let $\pi_{i}$ be the projection map $M \rightarrow G_{i}$. If some $\pi_{i}$ is not surjective, then $M$ lies in $\left(\prod_{j \neq i} G_{j}\right) \cdot \pi_{i}(M)$, which is contained in a member of $\mathcal{M}(G)$ under (1) of the definition above. Otherwise, all $\pi_{i}$ are surjective and we easily see that $M$ lies in a member of $\mathcal{M}(G)$ under (2) above.

By Proposition 2.3, there are only finitely many $G$-classes of subgroups in $\mathcal{M}(G)$ under (1) in the definition above. If the collection of subgroups under (2) is non-empty, then it consists of finitely many $G$-classes if $p=0$, and infinitely many classes if $p>0$, since in this case we can adjust the morphism $\phi$ by an arbitrary field twist.

Write $\mathcal{M}_{1}(G)$ for the collection of subgroups of $G$ under (1), so that $\mathcal{M}_{1}(G)$ consists of finitely many $G$-classes of subgroups.

If $H$ is a proper connected $G$-irreducible subgroup of $G$, then there is a sequence of subgroups

$$
H=H_{0}<H_{1}<\cdots<H_{s}=G
$$

such that for each $i, H_{i}$ is semisimple and $H_{i} \in \mathcal{M}\left(H_{i+1}\right)$. Write $\mathcal{M}_{0}(G)$ for the collection of $G$-irreducible subgroups $H$ for which there is such a sequence with $H_{i} \in \mathcal{M}_{1}\left(H_{i+1}\right)$ for all $i$. By Proposition 2.3 again, there are only finitely many $G$-classes of subgroups in $\mathcal{M}_{0}(G)$.

\section{Proof of Theorem 1}

Let $G$ be a connected semisimple algebraic group, and let $A$ be a connected $G$-irreducible subgroup of $G$. We prove that $A$ is contained in only finitely many subgroups of $G$.

The proof proceeds by induction on $\operatorname{dim} G$. The base case $\operatorname{dim} G=3$ is obvious. Clearly we may assume without loss that $Z(G)=1$. Write $G=G_{1} \cdots G_{r}$, a direct product of simple groups $G_{i}$, and let $\pi_{i}: G \rightarrow G_{i}$ be the $i$ th projection map.

LEMMA 3.1 If $H$ is a subgroup of $G$ containing $A$, then $H$ is closed and $H^{0}$ is semisimple.

Proof. Observe that $A^{H}=\left\langle A^{h}: h \in H\right\rangle$ is closed and connected, and hence $N_{\bar{H}}\left(A^{H}\right)$ is also closed. This normalizer contains $H$, hence contains $\bar{H}$. Thus $A^{H} \triangleleft \bar{H}^{0}$. By Lemma $2.1, \bar{H}^{0}$ is semisimple and $C_{G}(A)^{0}=1$. It follows that $A^{H}=\bar{H}^{0}$. Thus $\bar{H}^{0} \leqslant H \leqslant \bar{H}$. This means that $H$ is a union of finitely many cosets of $\bar{H}^{0}$, hence is closed, as required.

In view of this lemma, it suffices to show that the number of closed connected overgroups of $A$ in $G$ is finite. Suppose this is false, so that $A$ is contained in infinitely many connected subgroups of $G$. We shall obtain a contradiction in a series of lemmas.

By Lemma 2.1, $C_{G}(A)$ and $N_{G}(A) / A$ are finite. Recall the definitions in section 2 of the collections $\mathcal{M}(G)$ and $\mathcal{M}_{1}(G)$ of maximal connected subgroups of $G$.

LEMma 3.2 There exists $M \in \mathcal{M}(G)$ such that A lies in infinitely many $G$-conjugates of $M$.

Proof. First, if $A \leqslant M \in \mathcal{M}(G)$, then $M$ is semisimple by Lemma 2.1, and by induction $A$ has only finitely many overgroups in $M$. It follows that $A$ lies in infinitely many members of $\mathcal{M}(G)$.

We next claim that the overgroups of $A$ in $\mathcal{M}(G)$ represent only finitely many $G$-conjugacy 
classes of subgroups. For if not, there must exist $j, l$ such that $A$ lies in subgroups $G_{j, l}(\phi)$ for morphisms $\phi$ involving infinitely many different field twists. Since the high weights of composition factors of $L\left(G_{l}\right) \downarrow A$ are $\phi$-twists of those of $L\left(G_{j}\right) \downarrow A$ this implies that the highest weight of $A$ on $L(G)$ is arbitrarily large, a contradiction. This proves the claim, and the lemma follows.

From now on, let $M$ be the subgroup provided by Lemma 3.2.

LEMMA 3.3 M contains infinitely many $G$-conjugates of A, no two of which are $M$-conjugate.

Proof. By the previous lemma, $A$ lies in infinitely many conjugates of $M$; say $A$ lies in distinct conjugates $M^{g}$ for $g \in C$, where $C$ is an infinite subset of $G$. Let $g, h \in C$, so $A^{g^{-1}}$ and $A^{h^{-1}}$ lie in $M$; if these subgroups are $M$-conjugate, say $A^{g^{-1}}=A^{h^{-1} m}$ with $m \in M$, then $h^{-1} m g \in N_{G}(A)$. Letting $n_{1}, \ldots, n_{t}$ be coset representatives for $A$ in $N_{G}(A)$, we have $h^{-1} m g=a n_{i}$ for some $a \in A$ and some $i$. Thus $M^{g}=M^{\text {han }_{i}}$, so as $a \in M^{h}$, we have $M^{g}=M^{h n_{i}}$.

To summarize: fix $g \in C$; then if $h \in C$ is such that $A^{g^{-1}}$ and $A^{h^{-1}}$ are $M$-conjugate, we have $M^{h}=M^{g n_{i}^{-1}}$ for some $i$, so there are only finitely many such $h$. The lemma follows.

LEMMA $3.4 M \in \mathcal{M}_{1}(G)$.

Proof. Suppose not. Then there exist distinct $j, k \in\{1, \ldots, r\}$ and a surjective morphism $\phi: G_{j} \rightarrow G_{k}$, such that

$$
M=G_{j, k}(\phi)=G_{0} \cdot D_{j, k}
$$

where $G_{0}=\Pi_{i \neq j, k} G_{i}$ and $D_{j, k}=\left\{g \cdot \phi(g): g \in G_{j}\right\}$.

We may take it that $A \leqslant M$, so that each element of $A$ is of the form $a=a_{0} \cdot a_{j} \cdot \phi\left(a_{j}\right)$, where $a_{0} \in G_{0}, a_{j} \in G_{j}$. Since $M$ contains infinitely many $G$-conjugates of $A$, no two of them $M$-conjugate, it follows that $M$ contains infinitely many conjugates of the form $A^{g_{k}}\left(g_{k} \in G_{k}\right)$. If $a \in A$ is as above, then $a^{g_{k}}=a_{0} \cdot a_{j} \cdot \phi\left(a_{j}\right)^{g_{k}}$, so it follows that $\phi\left(a_{j}\right)^{g_{k}}=\phi\left(a_{j}\right)$ for all $a_{j} \in \pi_{j}(A)$. But this means that $g_{k} \in C_{G_{k}}\left(\pi_{k}(A)\right)$, which is finite; a contradiction.

LEMMA 3.5 There exists $M_{1} \in \mathcal{M}_{1}(M)$ such that $M_{1}$ contains infinitely many $G$-conjugates of $A$, no two of which are $M$-conjugate.

Proof. By Lemma 3.3, $M$ contains infinitely many $G$-conjugates of $A$, no two of which are $M$ conjugate. Call these conjugates $A^{g \lambda}(\lambda \in \Lambda)$, where $\Lambda$ is an infinite index set. For each $\lambda \in \Lambda$, there exists $M_{\lambda} \in \mathcal{M}(M)$ containing $A^{g_{\lambda}}$. Then infinitely many $M_{\lambda}$ are in $\mathcal{M}_{1}(M)$, since otherwise there exist $j, k$ such that $A^{g} \leqslant M_{j, k}(\phi)$ for morphisms $\phi$ involving infinitely many different field twists, which is impossible as in the proof of Lemma 3.2.

Since there are only finitely many $M$-classes of subgroups in $\mathcal{M}_{1}(M)$, infinitely many of the $M_{\lambda}$ lie in a single $M$-class of subgroups, with representative say $M_{1}$. Then $M_{1}$ contains infinitely many $G$-conjugates $A^{g_{\lambda} m_{\lambda}}\left(m_{\lambda} \in M\right)$, no two of which are $M$-conjugate.

Recall the definition of $\mathcal{M}_{0}(G)$ from section 2. Choose $N \in \mathcal{M}_{0}(G)$, minimal subject to containing infinitely many $G$-conjugates of $A$, no two of which are $N$-conjugate.

LEMMA 3.6 There are infinitely many distinct $G$-conjugates of A lying in $\mathcal{M}(N)$, no two of which are $N$-conjugate. 
Proof. Say $A^{g_{\lambda}}(\lambda \in \Lambda)$ are infinitely many conjugates of $A$ lying in $N$, no two of them $N$ conjugate. If the conclusion of the lemma is false, then for infinitely many $\lambda$, there is a subgroup $N_{\lambda} \in \mathcal{M}(N)$ such that $A^{g_{\lambda}} \leqslant N_{\lambda}$. As in the previous proof, infinitely many of these $N_{\lambda}$ are in $\mathcal{M}_{1}(N)$, of which there are only finitely many $N$-classes, so infinitely many $N_{\lambda}$ are $N$-conjugate to some $N_{1} \in \mathcal{M}_{1}(N)$. But then $N_{1}$ contains infinitely many $G$-conjugates of $A$ (namely $A^{g_{\lambda} n_{\lambda}}$ for some $n_{\lambda} \in N$ ), no two of which are $N$-conjugate, contradicting the minimal choice of $N$.

At this point we can obtain a contradiction. Write $N=N_{1} \cdots N_{k}$, a commuting product of simple factors $N_{i}$. By Lemma 3.6, there are infinitely many distinct $G$-conjugates $A^{g_{\lambda}}$ lying in $\mathcal{M}(N)$, no two of which are $N$-conjugate. As $\mathcal{M}_{1}(N)$ consists of only finitely many $N$-classes of subgoups, infinitely many of the $A^{g_{\lambda}}$ are in $\mathcal{M}(N) \backslash \mathcal{M}_{1}(N)$. Hence there exist $j, l$ such that infinitely many $A^{g}$ are of the form $N_{j, l}\left(\phi_{\lambda}\right)$, where $\phi_{\lambda}$ is a surjective morphism $N_{j} \rightarrow N_{l}$, and no two of these subgroups are $N$-conjugate. Then the morphisms $\phi_{\lambda}$ must involve infinitely many different field twists, which is a contradiction as usual, as it implies that the highest weight of $A$ on $L(G)$ (which is of course the highest weight of each conjugate $A^{g_{\lambda}}$ ) is arbitrarily large.

This completes the proof of Theorem 1 .

\section{Proof of Theorem 2}

Let $G$ be a simple algebraic group over $K$ in characteristic $p$, as in Theorem 2 (so that if $G=A_{n}$ then $p>n$ or $p=0$ ). We aim to construct a $G$-irreducible subgroup $A \cong A_{1}$.

LEMMA 4.1 The conclusion of Theorem 2 holds if $p=0$.

Proof. Suppose $p=0$. First consider the case where $G$ is classical. The irreducible representation of $A_{1}$ of high weight $r$ embeds $A_{1}$ in $S p_{r+1}$ if $r$ is odd, and in $S O_{r+1}$ if $r$ is even. Hence $S L_{n}, S p_{2 n}$ and $S O_{2 n+1}$ all have irreducible subgroups $A_{1}$. As for the remaining case $G=S O_{2 n}$, an $A_{1}$ embedded irreducibly in a subgroup $S_{2 n-1}$ is $G$-irreducible.

When $G$ is of exceptional type, but not $E_{6}$, it has a maximal subgroup $A_{1}$ (see [7]), and this is obviously $G$-irreducible; and for $G=E_{6}$, a maximal $A_{1}$ in a subgroup $F_{4}$ is $G$-irreducible (its connected centralizer in $G$ is trivial, so it cannot lie in any Levi subgroup).

In view of Lemma 4.1, we assume from now on that $p>0$.

LEMMA 4.2 The conclusion of Theorem 2 holds if $G$ is classical.

Proof. Assume $G$ is classical. If $G=A_{n}=S L_{n+1}$ then $p>n$ by hypothesis, so $G$ has a subgroup $A_{1}$ acting irreducibly on the natural $n+1$-dimensional $G$-module (with high weight $n$ ); clearly this subgroup does not lie in a parabolic of $G$.

Next, if $G=C_{n}=S p_{2 n}$, then $G$ has a subgroup $\left(S p_{2}\right)^{n}=\left(A_{1}\right)^{n}$, and we choose a subgroup $A \cong A_{1}$ of this via the embedding $1,1^{(p)}, 1^{\left(p^{2}\right)}, \ldots, 1^{\left(p^{n-1}\right)}$; then $A$ fixes no non-zero totally isotropic subspace of the natural module, hence lies in no parabolic of $G$. Similarly, if $G=D_{2 n}=$ $S O_{4 n}$, then $G$ has a subgroup $\left(S_{4}\right)^{n}=\left(A_{1}\right)^{2 n}$, and we choose $A \cong A_{1}$ in this via the embedding $1,1^{(p)}, \ldots, 1^{\left(p^{2 n-1}\right)}$.

Now let $G=D_{2 n+1}=S O_{4 n+2}$. Then $G$ has a subgroup $S O_{6} \times\left(S O_{4}\right)^{n-1} \cong A_{3} \times\left(A_{1}\right)^{2(n-1)}$, which contains a subgroup $\left(A_{1}\right)^{2 n}$ lying in no parabolic of $G$; choose $A \cong A_{1}$ in this $\left(A_{1}\right)^{2 n}$ via the embedding $1,1^{(p)}, \ldots, 1^{\left(p^{2 n-1}\right)}$ again.

Finally, for $G=B_{2 n}=S O_{4 n+1}$, choose $A \cong A_{1}$ in a subgroup $\left(S O_{4}\right)^{n}=\left(A_{1}\right)^{2 n}$ via the above embedding, while for $G=B_{2 n+1}=S O_{4 n+3}$ choose $A$ in a subgroup $S O_{3} \times\left(S_{4}\right)^{n} \cong\left(A_{1}\right)^{2 n+1}$. This completes the proof. 
Assume from now on that $G$ is of exceptional type. We choose our subgroup $A \cong A_{1}$ as follows. For $G=E_{8}, E_{7}, F_{4}$ or $G_{2}$, there is a maximal rank subgroup $\left(A_{1}\right)^{l}$ (where $l=8,7,4$ or 2 respectively), and we choose

$$
A<\left(A_{1}\right)^{l} \text {, via embedding } 1,1^{\left(p^{2}\right)}, 1^{\left(p^{4}\right)}, \ldots, 1^{\left(p^{2(l-1)}\right)} .
$$

For $G=E_{6}$ with $p>2$, there is a maximal rank subgroup $\left(A_{2}\right)^{3}$, and we choose

$$
A<\left(A_{2}\right)^{3}, \text { via embedding } 2,2^{\left(p^{2}\right)}, 2^{\left(p^{4}\right)} \text {. }
$$

Finally, for $G=E_{6}$ with $p=2$, take a subgroup $F_{4}$ of $G$, and a subgroup $C_{4}$ of that, generated by short root groups in $F_{4}$; now take $A<C_{4}$, embedded via the irreducible symplectic 8-dimensional representation $1 \otimes 1^{(2)} \otimes 1^{(4)}$.

LEMMA 4.3 (i) For $G \neq E_{6}, L(G) / L\left(A_{1}^{l}\right)$ restricts to $A$ as follows:

$G=E_{8}: 14$ distinct 4-fold tensor factors,

$G=E_{7}:$ seven distinct 4-fold tensor factors,

$G=F_{4}:$ one 4-fold factor and six distinct 2-fold factors,

$G=G_{2}: 1 \otimes 3^{\left(p^{2}\right)}(p \neq 2,3) ; 1 \otimes 1^{(9)} / 1 \otimes 1^{(27)}(p=3) ; 1 \otimes 1^{(4)} \otimes 1^{(8)}(p=2)$. Moreover, $L\left(A_{1}^{l}\right)$ restricts to $A$ as $2 / 2^{\left(p^{2}\right)} / \ldots / 2^{\left(p^{2(l-1)}\right)}$ if $p \neq 2$, and as $1^{(2)} / 1^{(8)} / \ldots / 1^{\left(2^{2 l-1}\right)} / 0^{l}$ if $p=2$.

In particular, the non-trivial composition factors of $L(G) \downarrow$ A are all distinct.

(ii) For $G=E_{6}(p \neq 2), L(G) / L\left(A_{2}^{3}\right)$ restricts to $A$ as $\left(2 \otimes 2^{\left(p^{2}\right)} \otimes 2^{\left(p^{4}\right)}\right)^{2}$; and $L\left(A_{2}^{3}\right)$ restricts to A as $2 / 2^{\left(p^{2}\right)} / 2^{\left(p^{4}\right)} / 4 / 4^{\left(p^{2}\right)} / 4^{\left(p^{4}\right)}$ if $p \neq 3$, and as $2 / 2^{\left(3^{2}\right)} / 2^{\left(3^{4}\right)} / 1 \otimes 1^{(3)} / 1^{\left(3^{2}\right)} \otimes 1^{\left(3^{3}\right)} / 1^{\left(3^{4}\right)} \otimes 1^{\left(3^{5}\right)} / 0^{3}$ if $p=3$.

(iii) For $G=E_{6}(p=2)$, letting $V_{27}=V_{G}\left(\lambda_{1}\right)$, we have

$$
V_{27} \downarrow A=1^{(2)} \otimes 1^{(4)} / 1^{(2)} \otimes 1^{(8)} / 1^{(4)} \otimes 1^{(8)} / 1^{(2)} / 1^{(2)} / 1^{(4)} / 1^{(4)} / 1^{(8)} / 1^{(8)} / 0^{3} .
$$

Proof. (i) For $G=E_{8}$, the restriction of $L(G)$ to a subsystem $D_{4} D_{4}$ is given by [4, 2.1]: it is $L\left(D_{4} D_{4}\right) / \lambda_{1} \otimes \lambda_{1} / \lambda_{3} \otimes \lambda_{3} / \lambda_{4} \otimes \lambda_{4}$. Now consider the restriction further to $A_{1}^{8}$. This is embedded as $\mathrm{SO}_{4} \cdot \mathrm{SO}_{4}$ in each $D_{4}$ factor, so the factor $\lambda_{1} \otimes \lambda_{1}$ of $L(G) \downarrow D_{4} D_{4}$ restricts to $A_{1}^{8}$ as a sum of 4-fold tensor factors, each of dimension 16. The normalizer $N_{G}\left(A_{1}^{8}\right)$ acts as the 3-transitive permutation group $A G L_{3}(2)$ on the eight factors, and the smallest orbit of this on 4-sets has size 14. It follows that $L(G) \downarrow A_{1}^{8}$ has at least 14 distinct 4-fold tensor factors. Since $14 \cdot 16+\operatorname{dim} A_{1}^{8}=\operatorname{dim} G$, these 14 modules comprise all the composition factors of $L(G) / L\left(A_{1}^{8}\right)$ restricted to $A_{1}^{8}$. Part (i) follows for $G=E_{8}$. The other types are handled similarly.

(ii) The restriction $L\left(E_{6}\right) \downarrow\left(A_{2}\right)^{3}$ is given by [4, 2.1], and (ii) follows easily.

(iii) We have $V_{27} \downarrow F_{4}=V_{F_{4}}\left(\lambda_{4}\right) / 0$, and $V_{F_{4}}\left(\lambda_{4}\right) \downarrow C_{4}=V_{C_{4}}\left(\lambda_{2}\right)$. Hence $V_{27} \downarrow C_{4}$ has the same composition factors as the wedge-square of the natural 8-dimensional $C_{4}$-module, minus one trivial composition factor. Now, to get the conclusion, calculate the composition factors of the $A_{1}$-module $\wedge^{2}\left(1 \otimes 1^{(2)} \otimes 1^{(4)}\right)$.

LEMMA 4.4 The subgroup A is G-irreducible.

Proof. First assume $G \neq E_{6}$. If $A<P=Q L$, a parabolic subgroup with unipotent radical $Q$ and Levi subgroup $L$, then the composition factors of $A$ on $L(Q)$ are the same as those on $L\left(Q^{\text {opp }}\right)$, 
the Lie algebra of the opposite unipotent radical. By the last sentence of Lemma 4.3(i), it follows that all composition factors of $A$ on $L(Q)$ must be trivial, whence from Lemma 4.3(i) we see that $\operatorname{dim} Q \leqslant l / 2$, which is impossible.

Now assume $G=E_{6}$ with $p \neq 2$. If $p \neq 3$ then $L(G) \downarrow A$ has no trivial composition factors, so $A$ cannot lie in a parabolic. Now suppose $p=3$. By Lemma 4.3(ii), $L(G) \downarrow A$ has two isomorphic 27-dimensional composition factors. If $A<Q L$ as above, then these factors must occur in $L(Q)+L\left(Q^{\mathrm{opp}}\right)$, and the only other possible composition factors in $L(Q)+L\left(Q^{\mathrm{opp}}\right)$ are trivial. Hence $\operatorname{dim} Q$ must be 27 or 28 . There is no such unipotent radical in $E_{6}$.

Finally, assume $G=E_{6}$ with $p=2$. Suppose $A<P=Q L$, with the parabolic $P$ chosen minimally. By minimality, $A$ must project irreducibly to any $A_{r}$ factor of $L^{\prime}$; since the irreducible representations of $A$ have dimension a power of 2 , it follows that the only possible such factors are $A_{3}$ and $A_{1}$. Consequently either $L^{\prime}=A_{3} A_{1}$, or $L^{\prime}$ lies in a subsystem $D_{5}$. If $L^{\prime}=A_{3} A_{1}$, then $A$ acts on the natural modules for $A_{3}, A_{1}$ as $1 \otimes 1^{(q)}, 1^{\left(q^{\prime}\right)}$ respectively, for some powers $q, q^{\prime}$ of 2. The restriction $V_{27} \downarrow A_{3} A_{1}$ is given by [4, 2.3], and it follows that $V_{27} \downarrow A$ has a composition factor $1 \otimes 1^{(q)} \otimes 1^{\left(q^{\prime}\right)}$ if $q \neq q^{\prime}$, and has two composition factors $1 \otimes 1^{(q)}$ if $q=q^{\prime}$. This conflicts with Lemma 4.3(iii). Therefore $L^{\prime} \neq A_{3} A_{1}$. The remaining possibilities for $L^{\prime}$ lie in a subsystem $D_{5}$. The irreducible orthogonal $A_{1}$-modules of dimension 10 or less have dimensions 4 and 8 , and do not extend the trivial module (see [1, 3.9]). It follows that $L^{\prime} \leqslant D_{4}$. Observe that $V_{27} \downarrow D_{4}=\lambda_{1} / \lambda_{3} / \lambda_{4} / 0^{3}$. Hence it is readily checked that no possible embedding of $A$ in $D_{4}$ gives composition factors for $V_{27} \downarrow A$ consistent with Lemma 4.3(iii).

This completes the proof of Theorem 2 .

By varying the field twists involved in the definitions of $A$ above, we obtain the following.

COROLlARY 4.5 Let $G$ be a simple algebraic group in characteristic $p>0$, and assume that $G \neq A_{n}$. Then $G$ has infinitely many conjugacy classes of $G$-irreducible subgroups of type $A_{1}$.

\section{Proof of Theorem 3}

Let $G$ be a connected semisimple algebraic group of rank $l$. The proof proceeds by induction on $\operatorname{dim} G$. The base case $\operatorname{dim} G=3$ is trivial. Let $X$ be a connected $G$-irreducible subgroup of $G$. By Lemma 2.1, $X$ is semisimple. Write $G=G_{1} \ldots G_{r}$ and $X=X_{1} \ldots X_{s}$, commuting products of simple factors $G_{i}$ and $X_{i}$. Without loss we can factor out the finite group $Z(G)$, and hence assume that $Z(G)=1$.

Suppose first that $X$ projects onto every simple factor $G_{i}$ of $G$. Say $X_{1}$ projects onto the factors $G_{1}, \ldots, G_{t}$. Identifying the direct product $G_{1} \ldots G_{t}$ with $G_{1} \times \ldots \times G_{1}$ ( $t$ factors), and replacing $X$ by a suitable $G$-conjugate, we can take

$$
X_{1}=\left\{\left(x^{\tau_{1}}, \ldots, x^{\tau_{t}}\right): x \in G_{1}\right\}
$$

where each $\tau_{i}=\gamma_{i} q_{i}$ with $\gamma_{i}$ a graph automorphism or 1 , and $q_{i}$ a Frobenius morphism or 1. For each $k$ let $S_{k}=\left\{i: q_{i}=q_{k}\right\}$, and define a corresponding subgroup $G_{S_{k}} \leqslant \prod_{i \in S_{k}} G_{i}$ by

$$
G_{S_{k}}=\left\{\prod_{i \in S_{k}} x^{\gamma_{i}}: x \in G_{1}\right\} \text {. }
$$

Then $X_{1}$ is a twisted diagonal subgroup of $G_{1}^{+}:=\prod_{S_{k}} G_{S_{k}}$. Repeating this construction for each 
simple factor $X_{i}$ of $X$, we obtain a subgroup $G_{1}^{+} \ldots G_{s}^{+}$of $G$ containing $X$ as a twisted diagonal subgroup. There are only finitely many such subgroups $G_{1}^{+} \ldots G_{s}^{+}$in $G$. Hence if we include the conjugacy classes of these subgroups in our collection $\mathcal{C}$, we have the conclusion of Theorem 3 in this case.

Now suppose $X$ does not project onto some factor, say $G_{1}$, of $G$. Then there exists a maximal connected subgroup $M_{1}$ of $G_{1}$ such that $X \leqslant M_{1} G_{2} \cdots G_{r}$. By Proposition 2.3, up to $G_{1}$-conjugacy there are only finitely many possibilities for $M_{1}$. Since $M_{1} G_{2} \ldots G_{r}$ is a semisimple group of dimension less than $\operatorname{dim} G$, the result now follows by induction.

\section{Acknowledgements}

The second author acknowledges the support of the Swiss National Science Foundation grant MHV 21-65839.

\section{References}

1. H. H. Andersen, J. Jorgensen, and P. Landrock, The projective indecomposable modules of SL $\left(2, p^{n}\right)$, Proc. London Math. Soc. 46 (1983), 495-528.

2. F. Bien and A. Borel, Sous-groupes epimorphiques de groupes linéaires algébriques I, C.R. Acad. Sci. Paris 315 (1992), 649-653.

3. A. Borel and J. Tits, Éléments unipotents et sousgroupes paraboliques de groupes réductifs, Invent. Math. 12 (1971), 95-104.

4. M. W. Liebeck and G. M. Seitz, Reductive subgroups of exceptional algebraic groups, Mem. Amer. Math. Soc. 121 (1996).

5. M. W. Liebeck and G. M. Seitz, The maximal subgroups of positive dimension in exceptional algebraic groups, Mem. Amer. Math. Soc. to appear.

6. M. W. Liebeck and D. M. Testerman, Some epimorphic subgroups of simple algebraic groups, to appear.

7. G. M. Seitz, Maximal subgroups of exceptional algebraic groups, Mem. Amer. Math. Soc. 90 (1991).

8. J.-P. Serre, Moursund Lecture Notes, University of Oregon Mathematics Department, 1998. 